\title{
SI LOS ADMINISTRADORES PENSARAN COMO DISENADORES
}

JeAnNe M. LiedtKa

\section{RESUMEN}

El diseño es un concepto muy discutido ahora en el mundo de los negocios y es considerado por muchos como el "arma secreta" que apoyará a las empresas en la competencia para encontrar un lugar destacado en el ámbito lleno de argucias, que seguramente se vivirá en el siglo XXI. El artículo se pregunta "qué significaría para la estrategia de negocios que los administradores tomáramos en serio la idea del diseño".

El análisis de diez interesantes procesos de diseño motivan respuestas inteligentes a este interrogante. El Teatro de la Ópera de Sydney, creado por Jørn Utzon, Premio Pritzker en el 2003 y que es hoy un motivo de orgullo para la ciudad. El Museo Guggenheim en Bilbao, creado por Frank Gehry, con una gran intuición sobre lo que atraería a los vascos. El vestidito negro (little black dress), diseñado por Coco Chanel en la década de los 20, cuya sencillez constituyó un acontecimiento. El puente Golden Gate de San Francisco, que se ha transformado en símbolo de la ciudad. Una hermosa pintura realizada por Picasso a los 14 años, la Primera Comunión, elaborada de manera convencional, comparada con la innovadora factura del Guernica, que conmemora el bombardeo nazi del pueblo vasco. La "visionaria" compañía ideada por Ingvar Kamprad, IKEA, que transforma el negocio de los muebles. La innovación en los diseños urbanos logrados gracias el proceso charrette de los proyectos del Nuevo Urbanismo de la Comunidad Seaside en Florida. La concepción del Parque Central de Nueva York, asignada por concurso a Frederick Law Olmsted y Calvert Vaux y que dio esa "sensación campestre" a la ciudad ombligo del mundo. La Universidad de Virginia, a la cual dedicó Thomas Jefferson los últimos años de su vida, y que generó una "comunidad" donde "los estudiantes trabajan como socios" para establecer el diálogo que "requiere la democracia". Y, finalmente, la inacabada catedral de La Sagrada Familia en Barcelona, ideada por Antoni Gaudí como una "Biblia de piedra".

La autora indica que "las diez historias de diseño relatadas aquí tratan de posibilidades hechas realidad" y que cada una de estas grandes creaciones encarna una virtud necesaria para que una estrategia inteligente y creadora de negocios sea capaz de innovar en este siglo.

\section{PALABRAS CLAVE}

Diseño, estrategia, administradores, administración, invención, concepciones estratégicas.

\section{ABSTRACT}

The design is a very well discussed concept nowadays in the business world and is consi- 
dered by many as the "secret weapon" that will support the enterprises in the competence to find a prominent place in the environment full of cunning arguments which the XXI century will live for sure. The article questions "what would it mean for the business strategy that administrators took seriously the idea of design".

The analysis of ten interesting design processes motivates intelligent responses to this questioning. The Theater of Sydney Opera, created by Jørn Utzon, Pulitzer Prize in 2003 and that today is the reason of pride for the city. The Guggenheim Museum in Bilbao, created by Frank Gehry, with a great intuition about what would attract the Basque. The little black dress designed by Coco Chanel in the 1920s whose simplicity was an event. The Golden Gate Bridge in San Francisco which has been transformed in city symbol. A beautiful picture performed by Picasso when he was 14, the First Communion elaborated conventionally, compared to the innovative making of the Guernica that commemorates the nazi bombing of the Basque people. The "visionary" company devised by Ingvar Kamprad, IKEA that transforms the furniture business.

The innovation in urban designs reached thanks to the charrette process of the New Urbanism of the Seaside Community in Florida. The conception of Central Park in New York assigned by contest to Frederick Law Olmsted and Calvert Vaux and which gave that "countryside sensation" to the center city of the world. The University of Virginia, to which Thomas Jefferson devoted the last days of his life, and that brought about a "community" where "students work as partners" to establish the dialogue that "the democracy requires". And finally, the unfinished cathedra of the Sacred Family in Barcelona, devised by Antonio Gaudi as a "Stone Bible".

The author points out that the "ten design stories told here are about possibilities made true" and that each of these great creations embodies a necessary virtue for one intelligent and creative strategy of business is able to innovate in this century.

\section{INVIERNO 2005 • CONFERENCIAS BATTEN}

Una discusión acerca de la innovación, el espíritu emprendedor, y la transformación corporativa.

El diseño es un tema muy discutido en el mundo de los negocios de hoy. De hecho, ha sido llamado el "arma secreta" para la competencia en el siglo veintiuno. Pero, debajo de toda la exageración, tenemos que preguntarnos qué significaría para la estrategia de negocios que los administradores tomáramos en serio la idea del diseño. ¿Qué tal si tratáramos de pensar en la forma que lo hacen los diseñadores? Habiendo estu- diado durante la última década cómo los diversos tipos de diseñadores trabajan y crean, ofrezco las siguientes diez sugerencias como punto de partida en el diálogo.

Si tomáramos en serio la metáfora del diseño...

1. Nos daríamos cuenta que el diseño de la estrategia de negocios es cosa de invención. Los estrategas, pese a toda su retórica acerca de "arte y ciencia" de la administración, más que en otra cosa, han puesto la atención en la búsqueda analítica de la única estrategia correcta. Tomar en serio la 
metáfora del diseño, sin embargo, significa reconocer en primer término, la diferencia entre lo que hacen los científicos y lo que hacen los diseñadores. Mientras los científicos investigan el hoy para descubrir explicaciones para lo que ya está, los diseñadores inventan el mañana para crear algo que no está.

Nos importa la estrategia porque queremos que el futuro sea distinto al presente. Pero los futuros impactos raramente se descubren a través del análisis. Estos son, como dijo Walt Disney, "creados primero en la mente y luego en la actividad". Esto no niega un importante papel al análisis, pero sí subordina el análisis al proceso de la invención.

Ofrezco, a manera de ejemplo de la tensión existente entre invención y análisis, el Teatro de la Ópera de Sydney, cuyo diseñador, Jørn Utzon, fue premiado con el más alto galardón de la arquitectura, el Premio Pritzker, en 2003. Es difícil imaginar ahora una Australia sin el Teatro de la Ópera de Sydney, pero es bastante posible que esta impactante invención nunca hubiera sido construida si los estimativos iniciales para el proyecto hubieran sido precisos. En 1957, cuando fue seleccionada la propuesta de Utzon, los contadores estimaron que el proyecto tomaría cinco años para completarse y costaría \$7 millones. En realidad, demoró 14 años y costó más de $\$ 100$ millones. John G. Lowe, quien registró la historia del teatro de la ópera, cita a Ove Arup, un ingeniero

\section{RESEÑA AUTOR:}

Jeanne M. Liedtka, directora ejecutiva del Batten Institute y Profesora Asociada de Investigación Johnson and Higgins en Administración de Empresas en el Darden School de la Universidad de Virginia. Se puede comunicar con ella en liedtkaJ@darden.virginia.edu. que colaboró con Utzon en el proyecto: "Si la magnitud de la tarea se hubiera apreciado por completo... el Teatro de la Ópera nunca se hubiera construido. Y el hecho de que no fuera conocido... fue una de las circunstancias inusuales que hicieron posible el milagro". Gracias a Dios que los contadores fallaron en su análisis.

2. Reconoceríamos la supremacía de la persuasión. Si la estrategia es en realidad una invención, sólo una historia entre muchas acerca del futuro, entonces siempre es discutible. Los líderes deben, por tanto, persuadir a otros de la gran sabiduría y superioridad de la historia que han elegido. Ellos deben, de hecho, hacer seductora la historia; en la venta de su estrategia, ellos deben, para decirlo de manera clara, tratar a los empleados como amantes en lugar de prostitutas.

No es fácil intentar convencer a las personas para que compartan una imagen del futuro. Después de todo, las estrategias en la mayoría de las industrias de hoy día, llaman a las personas a comprometerse con algo totalmente nuevo y diferente, a dar un paso para alejarse de la seguridad de lo que siempre ha funcionado en el pasado. No es una venta fácil, incluso para los líderes más veteranos. Persuadir a otros de compartir su visión, al igual que entrar en una nueva relación, funciona mejor cuando uno hace una invitación en lugar de dar una orden.

Los diseñadores entienden esto. Los arquitectos exitosos, por ejemplo, saben que para que sus grandiosos edificios sean construidos, ellos deben persuadir a los clientes para que los paguen, y que esto requiere ayudarles a los clientes a visualizar el edificio. De hecho, mientras más 
ingenioso sea el arquitecto, más crítica será la habilidad de evocar la imagen para el cliente y para lo que puede ser un público muy escéptico.

Cuando Frank Gehry comenzó a esbozar lo que se convertiría en el Museo Guggenheim en Bilbao, España, él ya tenía una fuerte intuición de lo que atraería a una audiencia vasca muy tradicional a su creación asombrosamente inventiva. Gehry explica su método: "Uno trae ciertas cosas a la mesa... los vascos, su deseo de usar la cultura, de traer la ciudad al río. Y la sensación industrial...”.

El crítico de arquitectura que escribe en Los Angeles Times, Nicolai Ouroussoff describe el resultado:

"Gehry ha logrado lo que no hace mucho parecía imposible para la mayoría de los arquitectos: la invención de formas arquitectónicas radicalmente nuevas que, sin embargo, hablan al hombre de la calle. Bilbao se ha convertido en un punto de peregrinación para aquellos quienes, hasta ahora, tenían poco interés en la arquitectura. Los fines de semana llegan parejas vascas de clase trabajadora con niños a bordo. Las élites culturales se desvían de sus trayectos de vuelo normales para poder decir a sus amigos que ellos también han visto el edificio en persona."

El Guggenheim de Gehry persuade y seduce conectando el pasado de los vascos y señalando un nuevo futuro. Así es como las estrategias se vuelven poderosas y persuasivas: le muestran a una organización su futuro sin olvidar su pasado. Nos cuentan qué podemos conservar como también qué debemos perder.
3. Valoraríamos la sencillez. Piense en un objeto que usted adora. Lo más probable es que sea suficientemente complejo para realizar bien su función, pero no más complejo de lo necesario. En otras palabras, es una solución elegante. Ningún diseño ejemplifica mejor la sencillez y elegancia que el vestidito negro de Channel (little black dress). El más llamativo aspecto del vestidito negro, diseñado por Coco Chanel en los años 20, es su sencillez. El vestido no se excede en prescribir o adornar sino que ofrece en su lugar un lienzo negro, que su portadora ajusta a la función en mano: agregue perlas y tacones altos para mayor elegancia; una bufanda de color fuerte y zapatos de tacón bajo para mayor informalidad. Las posibilidades no tienen fin, haciendo del vestido la prenda más funcional del vestuario de una mujer. Pero el vestidito negro va más allá de la mera funcionalidad para lograr la elegancia: no le falta nada esencial y no contiene nada extraño.

¿Qué tal si usáramos el vestidito negro como modelo de una estrategia de negocios? Terminaríamos con estrategias que no serían incomprensibles ni banales ni obvias a todos exceptuando a sus creadores. Estas olvidarían las modas y se enfocarían en elementos perdurables, incorporando una versatilidad y apertura que invitaría a sus "portadores" a agregar adornos para la ocasión del momento. Lo más importante tal vez sería que hicieran enfasis en nuestros puntos positivos a la vez que reconocerían nuestras fallas - todo al servicio de ofrecernos la esperanza de un mejor (y más esbelto) mañana.

4. Buscaríamos inspirar. Uno de los hechos más tristes del estado del diseño 
de negocios es el grado en el cual nos conformamos con la mediocridad. Ni siquiera intentamos atraer a nuestra audiencia en el campo emocional, mucho menos inspirar. Sin embargo, la diferencia entre los grandes diseños y los que apenas están bien es la manera como los primeros nos llaman a algo más grande.

Considérense las diferencias entre el puente de la Bahía de San Francisco y el Golden Gate. El Puente de la Bahía ofrece un camino por encima del agua. El Puente Golden Gate también hace eso, pero también transporta, simboliza y encanta. Al igual que el Teatro de la Ópera de Sydney, se ha convertido en un icono de la tierra que ocupa. ¿Cuántas de nuestras estrategias de negocios son como el Puente Golden Gate? Muy pocas.

5. Primero, nos volveríamos maestros en las habilidades básicas. Cada uno de los diseños que hemos mirado hasta ahora es inventivo, persuasivo, elegante, e inspirador. No obstante, todos tienen éxito porque también funcionan bien, y hacen esto debido a la maestría en cuanto a elementos técnicos. Las cubiertas en forma de vela del Teatro de la Ópera de Sydney demandaron ingeniería experta. La ondulante fachada exterior cubierta de titanio del Guggenheim de Bilbao sólo fue posible gracias a sofisticados modelos de computador. Y el vestidito negro funcionó porque Chanel fue pionera en un tejido sintéticojersey-que fluía en lugar de colgar.

Si uno examina la pintura de 1895 Primera Comunión, verá evidencia de esta técnica extraordinaria; en particular, las capas de blanco en el vestido de la joven, son asombrosas. ¿Quién era el artista?
Pablo Picasso, quien, a los 14 años, claramente había logrado maestría en el arte convencional. Considere ahora Guernica, que Picasso pintó en 1937 en memoria del bombardeo nazi del pueblo vasco. Hay poco de convencional en esta pintura, considerada una de las declaraciones antibélicas más poderosas del arte moderno. Picasso, quien para entonces era reconocido como uno de los artistas más influyentes del siglo veinte, había ido más allá de la técnica convencional, usando su maestría para impulsar las fronteras del arte.

6. Aprenderíamos a experimentar. ¿Cómo va uno de la maestría a la brillantez? ¿Desde la competencia técnica a la verdadera innovación? Experimentando. Algunos experimentos de diseño se llevan a cabo en la mente-piense en el proceso de planeación estratégica, en el cual los estrategas imaginan y ensayan nuevos futuros -y algunos encuentran su expresión en prototipos físicos. Algunos experimentos se realizan incluso en el mundo real, y aquí ofrezco mi única historia de diseño tomada del mundo de los negocios: IKEA. Cuando el visionario fundador de la compañía, Ingvar Kamprad, comenzó, sólo tenía una idea general de lo que se convertiría en la visión revolucionaria de IKEA respecto del negocio de muebles. Casi todos los elementos del ahora legendario modelo de negocios de IKEA — salas de exhibición y catálogos, muebles desarmables en empaques planos, y transporte y ensamblaje por parte del cliente- surgieron a lo largo del tiempo a partir de respuestas experimentales a problemas urgentes. El transporte por parte del cliente, por ejemplo, se volvió un elemento central de la estrategia de IKEA
Considérense las diferencias entre el puente de la Bahía de San Francisco $y$ el Golden Gate. El Puente de la Bahía ofrece un camino por encima del agua. El Puente Golden Gate también hace eso, pero también transporta, simbolizay encanta. Al igual que el Teatro de la Ópera de Sydney, se ha convertido en un icono de la tierra que ocupa. ¿Cuántas de nuestras estrategias de negocios son como el Puente Golden Gate? Muypocas. 
casi por accidente, cuando los clientes frustrados entraban a la bodega porque no había suficientes empleados para ayudarlos. El gerente del almacén se dio cuenta de las ventajas de la iniciativa de los clientes y sugirió que la idea se volviera permanente.

7. Seríamos más incluyentes en nuestras conversaciones estratégicas. La imagen del genio solitario trabajando en su taller es tan mítica en el arte, arquitectura y ciencia como lo es en los negocios. El diseño nos enseña acerca del valor de incluir múltiples perspectivas en el proceso de diseñovolviendo el proceso, una conversación. Mientras más complejo sea el reto de diseño, mayores los beneficios de múltiples voces y perspectivas.

Considérese, por ejemplo, el muy complejo, y político, proceso de la planeación urbana. Me gustaría considerar en particular, el Nuevo Movimiento Urbanista, el cual surgió de las experiencias de los urbanizadores y arquitectos de la innovadora Comunidad Seaside en Florida. Lo que diferencia al Nuevo Urbanismo de otros movimientos arquitectónicos es su énfasis en la amplia participación. Esta participación toma la forma de una charrette ${ }^{1}$, una conversación de diseño interactiva con larga tradición en el arte y arquitectura. Derivada de la palabra francesa que significa "pequeña carreta," las charrettes fueron usadas en la primera escuela de arquitectura formal, la École des Beaux Arts en París, en el siglo diecinueve. A medida que los estudiantes progresaban de un nivel a otro, ponían sus proyectos en pequeños carritos, sobre los cuales los estudiantes saltaban para hacer sus toques finales.

El proceso charrette usado en proyectos del Nuevo Urbanismo se basa en cuatro principios: involucre desde el comienzo a todos los que puedan construir, usar, vender, aprobar, o bloquear el proyecto; trabaje en forma simultánea e interdisciplinaria (arquitectos, planificadores, ingenieros, economistas, expertos en el mercado, ciudadanos, funcionarios públicos); trabaje en ciclos cortos de retroacción; y trabaje en los detalles.

El charrette, creo yo, ofrece una poderosa alternativa al proceso tradicional de planeación estratégica invitando a participar a todo el sistema e incluyendo conocimiento local en el diálogo.

8. Aprenderíamos a hablar de modo diferente. Es obvio que no basta simplemente con juntar a una variedad de personas. Para producir diseños superiores, debemos cambiar la forma en que hablamos entre nosotros. La mayoría de nosotros aprende a hablar en contextos de negocios como si estuviéramos en un debate, defendiendo una posición. No obstante, dentro de un grupo diverso, es más probable que el debate lleve a empates que a avances. Los avances surgen de hacer nuevas preguntas, no de debatir soluciones existentes. Vienen de reexaminar lo que damos por descontado.

Tómese como ejemplo el diseño del Parque Central de Nueva York. En 1857, se llevó a cabo el primer concurso de diseño paisajista público en el país para seleccionar el plan para este parque. Entre todas las propuestas, sólo una-preparada por Frederick Law Olmsted y Calvert Vaux-

1 En español carreta. Posiblemente tiene que ver con el significado de conversación o discurso que se le da en Colombia. 
llenó todos los requerimientos de diseño. El reto mayor-que se permitiera el paso del tráfico vehicular de la ciudad sin dañar la sensación campestre del parque-había sido considerado como imposible de cumplir por parte de todos los demás diseñadores. Olmsted y Vaux fueron exitosos al dejar de suponer que el parque era un espacio en dos dimensiones. En su lugar, ellos imaginaron el parque en tres dimensiones, y hundieron cuatro carreteras ocho pies debajo de su superficie.

9. Trabajaríamos de atrás hacia adelante. A la mayoría de los administradores se les enseña una metodología directa para la solución de problemas: defina el problema, identifique varias soluciones, analice cada una, y escoja una. Los diseñadores comienzan al final de este proceso, como en la famosa advertencia de Stephen Covey, logrando claridad acerca de los resultados deseados del diseño y luego trabajando de atrás hacia adelante.

Me gustaría ilustrar este método con una historia que es cercana. Thomas Jefferson, quien incluyó la educación entre sus muchas pasiones e intereses, dedicó la última década de su vida a la fundación de la Universidad de Virginia. Para Jefferson, el vínculo entre democracia y educación era claro: sin una población educada, no había esperanza de proteger la naciente democracia que él y los demás padres de la patria habían creado trabajando tan duramente. La universidad de Jefferson produciría graduados con mente abierta, por tanto necesitaría diferenciarse en muchas formas de las instituciones educativas dominantes. Ésta sería una comunidad donde la facultad y los estudiantes trabajarían como socios para crear un diálogo que produciría el tipo de aprendizaje que la democracia requiere. El típico gran edificio central, como aquel en el que Jefferson había vivido, estudiado, y trabajado en el College of William and Mary, sería reemplazado por una colección de edificios más pequeños. Esta "aldea académica” rodeada de jardines sería una comunidad del aprendizaje donde los estudiantes tendrían libertad sin precedentes tanto en la elección del currículo como en gobernar sus propios comportamientos.

El genio de Jefferson, para el observador moderno, puede parecer que yace en la belleza de la arquitectura que creó. En realidad, él tomó mucha de su inspiración arquitectónica directamente del arquitecto italiano del siglo dieciséis, Palladio. El verdadero genio de Jefferson yace en el poder del espacio que él creó y su habilidad para evocar de manera tan vívida el objeto para el cual fue diseñado.

10. Comenzaríamos el diálogo con posibilidades. El gran diseño, se ha dicho, ocurre en la intersección entre restricción, contingencia, y posibilidad - elementos que son centrales en la creación de diseños innovadores, elegantes, y funcionales. Sin embargo, importa mucho dónde se comienza. En los negocios, hemos tendido a comenzar la conversación estratégica con restricciones: las restricciones de presupuestos, de la facilidad en la implementación, del foco en ganancias trimestrales que dicta Wall Street. Como resultado, obtenemos diseños para mañana que simplemente se ajustan al hoy. El gran diseño inevitablemente comienza con la pregunta: “QQué tal si cualquier cosa fuera posible?” Después de todo, si la estrategia es una
El gran diseño, se ha dicho, ocurre en la intersección entre restricción, contingencia, $y$ posibilidadelementos que son centrales en la creación de diseños innovadores, elegantes, $y$ funcionales. 
invención, producto de nuestras imaginaciones, y nuestras suposiciones restringen lo que podemos imaginar, entonces remover las suposiciones que surgen de creer en esas restricciones es la tarea número uno.

Para nuestro último ejemplo de diseño, nos vamos a una de mis ciudades preferidas, Barcelona, y examinamos la historia de su gran catedral inconclusa, La Sagrada Familia, diseñada por Antoni Gaudí.

Gaudí tenía sólo 32 años en 1884 , cuando fue nombrado arquitecto principal de la iglesia conocida como la "Catedral de los Pobres," la cual sería construida por completo con donaciones. Desde el comienzo, Gaudí vislumbró la catedral que quería crear - una "Biblia en piedra," un interior encumbrado que evocaba un bosque y un exterior con torres que alcanzaban los cielos. Gaudí resolvió diseñar su catedral como si cualquier cosa fuera posible, aunque las restricciones a las que se enfrentó fueron numerosas y aparentemente insuperables. Gaudí eligió ignorar las restricciones usuales de tiempo y dinero. "Mi cliente no tiene prisa," era su respuesta a los escépticos quienes dudaban de que la iglesia se fuera a terminar algún día. Cuando los fondos se hicieron demasiado escasos para seguir construyendo, él regresó al diseño, construyendo modelos de yeso cada vez más detallados y saliéndose de su papel de arquitecto-constructor para conseguir fondos personalmente.

Las restricciones reales impuestas por los materiales y técnicas de construcción disponibles en ese tiempo eran imposibles de ignorar para Gaudí. Debido a que el mundo natural servía como una de sus fuentes primarias de inspiración en todos sus diseños, Gaudí aspiraba a crear espacios encumbrados con luz natural y se encontró profundamente impedido por la necesidad de muros interiores de carga y vigas rectas. Sin el conocimiento matemático y técnicas de modelación a disposición, hoy en día, la física de la construcción de la catedral era también un reto a medida que Gaudí buscaba evitar los enormes arcos y arbotantes comunes a las catedrales medievales.

Para poder trabajar sobreponiéndose a estas restricciones, Gaudí buscó nuevas herramientas y técnicas. Él encontró dos herramientas poco usadas en esos días en Barcelona, que se convertirían en el cimiento de su trabajo. El primero fue el arco en catenaria, un arco simple cuya forma podía simularse suspendiendo una cadena al revés. Gaudí pudo calcular las exigencias de carga impuestas a las enormes torres de la catedral suspendiendo pequeñas bolsas de arena desde la cadena invertida para imitar el peso que las torres tendrían que portar. Esto creó un modelo perfecto (¡aunque al revés!) de las posibles formas y dimensiones que una torre verdadera podía asumir. Modelos de computador hechos con las torres de Gaudí demuestran la sorprendente precisión de este método.

La segunda herramienta que él descubrió fue un material nuevo: cemento. Combinado con vigas de hierro, columnas de ladrillo o piedra, y un nuevo aprovechamiento del cerrado de techos, el cemento permitía a los muros exteriores portar la mayor parte del peso del techo, lo que dio a Gaudí la libertad de diseño interior que añoraba.

Gaudí murió a la edad de 74 años (atropellado, camino a misa, por un tranvía en Barcelona) con su catedral parcialmente terminada. Diez años más tarde, llegó la 
guerra civil española a la ciudad y frenó la construcción. Unos amotinados quemaron su taller, destruyendo todos sus planos y archivos. Afortunadamente, los modelos de yeso sobrevivieron al incendio y son usados hoy para guiar la fase final de la construcción de la catedral. Se espera terminar la construcción dentro de los próximos 20 años.

Todas las diez historias de diseño relatadas aquí tratan de posibilidades hechas realidad, algunas de ellas con mucho en contra. Diseños de este tipo son difíciles de lograr. Para poderlo hacer, debemos primero aspirar a lograrlos, retando la mediocridad de muchos de los diseños de hoy. También debemos aprender nuevas destrezas - la maestría de las tecnologías básicas y la habilidad para persuadir, hablar de manera distinta, experimentar. Finalmente, debemos dar la bienvenida a los nuevos procesos- procesos que invitan a un conjunto de perspectivas más diversas al diálogo estratégico, que trabajan de atrás para adelante a partir de un sentido claro de los resultados que queremos crear, diálogos que comienzan con posibilidades. El tipo de diseños ejemplares que hemos examinado aquí rara vez se logran incluso en el diseño, mucho menos en los negocios. Pero como sabemos todos, lo más difícil de hacer es lo que más vale la pena hacer.

Batten Briefings, Volumen 4, Número 1, invierno 2005. Traducido y publicado con el permiso del Instituto Batten en el Darden Graduate School of Business Administration, University of Virginia.

Batten Briefings, Volume 4, Number 1, Winter 2005. Translated and published with permission by the Batten Institute at the Darden Graduate School of Business Administration, University of Virginia. 